Itinéraires Itinéraires

Littérature, textes, cultures

\title{
Stéréotypes, écrits coloniaux et postcoloniaux : le cas de l'Algérie
}

Fatima Zohra Lalaoui-Chiali

\section{OpenEdition}

Journals

Édition électronique

URL : http://journals.openedition.org/itineraires/2125

DOI : $10.4000 /$ itineraires. 2125

ISSN : 2427-920X

Éditeur

Pléiade

Édition imprimée

Date de publication : 1 mai 2010

Pagination : 157-171

ISBN : $978-2-296-11224-7$

ISSN : $2100-1340$

\section{Référence électronique}

Fatima Zohra Lalaoui-Chiali, «Stéréotypes, écrits coloniaux et postcoloniaux : le cas de l'Algérie », Itinéraires [En ligne], 2010-1 | 2010, mis en ligne le 01 mai 2010, consulté le 30 avril 2019. URL : http:// journals.openedition.org/itineraires/2125; DOI : 10.4000/itineraires.2125

\section{(C) $(\oplus \Theta$}

Itinéraires est mis à disposition selon les termes de la licence Creative Commons Attribution - Pas d'Utilisation Commerciale - Pas de Modification 4.0 International. 


\section{Stéréotypes, écrits coloniaux et postcoloniaux : le cas de l'Algérie}

\section{Abstract}

The purpose of this article is to understand how a complete system of representation evolved from a stereotype of the Sarracen-Arab and effectively perpetuated down to the present time a negative image of the Arab in general, and more specifically of the Algerian. This image has served as a core for the construction of a whole ideological discourse which prevents alterity from functioning along the lines of respect and tolerance.

Keywords : Algeria, colonial and postcolonial writings, Sarracens, stereotype, systems of representation

Mots clés : Algérie, écrits coloniaux et postcoloniaux, Sarrasins, stéréotype, systèmes de représentation

\section{Introduction}

Le roman colonial s'inscrit dans un processus historique lié aux conjonctures politiques et sociales qui, tout en l'influençant, le condamnent à ne demeurer que le résultat d'une circonstance. Pourtant, la lecture, même lorsqu'elle concerne les textes littéraires, est souvent perçue comme un acte socioculturel sur lequel s'exercent d'autres influences que les attentes spécifiquement littéraires. Concernant le roman colonial algérien, il serait intéressant de voir de quelle manière la littérature a construit certains stéréotypes et dans quels buts. Comment des images comme celle du Sarrasin ${ }^{1}$ (envahisseur arabe) ont-elles évolué, se sont-elles transformées pour devenir

1. Le mot Sarrasin/Sarrazin dérivé du latin Sarracenus et du grec Sarakenos fut utilisé par les Romains pour désigner les envahisseurs dangereux, venus d'Arabie, qui s'étaient installés à leur frontière orientale. 
celles des barbaresques (un mélange de sept races) puis se figer pour devenir le/les stéréotype(s) de l'Arabe ou de l'indigène algérien?

\section{La représentation du Sarrasin au Moyen Âge}

Dès cette époque, on a considéré le Sarrasin comme l'ennemi des Francs. Cette appellation désignait alors aussi bien l'Arabe, le Maghrébin que le Basque, le Normand ou le Hongrois. Ce temps fut vite révolu puisque, durant une période de ferveur religieuse, on ne désignait du nom de Sarrasins - l'appellation devenant péjorative - que les seuls musulmans. Peu après on s'autorisera à dire que Roland, symbole du preux chevalier, a été battu par des guerriers sarrasins (synonymes de musulmans) et non par des Basques comme le rapporte l'histoire relatant la bataille de Roncevaux.

Le Sarrasin sera diabolisé; quant à l'assimilation de l'islam au paganisme, écrivains ecclésiastiques et chroniqueurs de l'époque n'hésitent pas à attribuer aux musulmans le comportement idolâtre des païens de l'Antiquité. Dans La Chanson de Roland, ils (les musulmans) :

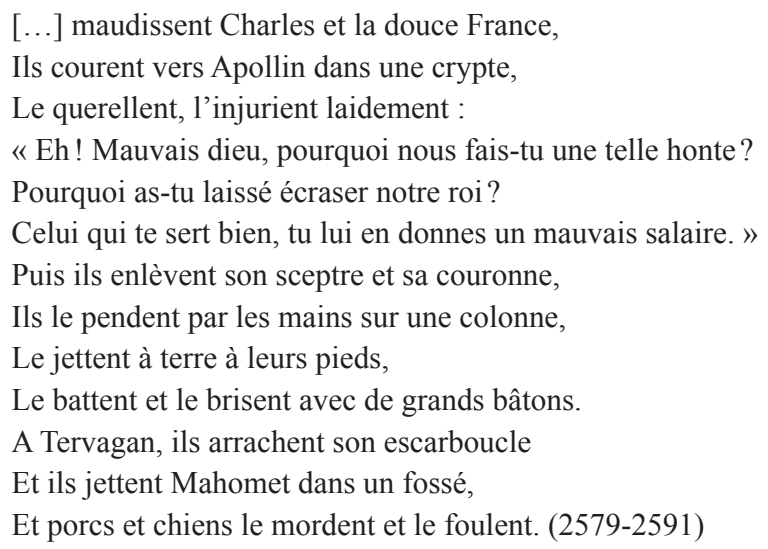

Les personnages de la chanson de geste ${ }^{2}$ se répartissent entre les deux côtés d'une ligne - qui sépare les « bons » des « méchants ». Les héros chevaleresques incarnent toutes les vertus : l'honneur, la fidélité, le courage et la dextérité au combat. Ils sont jeunes, beaux, et blancs. Ils tuent mais cette

2. Dans une réécriture en français moderne de La Chanson de Rolland, Marsile, symbole du Sarrasin est le roi maure qui tient tête aux armées de Charlemagne. Il est sauvage mais résolu, féroce, typé et craint. Il est encore coléreux, sans cœur, orgueilleux et prudent. Son armée est composée de « Nègres ». Pour les désigner, les mots « païen » et ses dérivés interviennent cinquante fois, les mots « Sarrasins » (huit fois), « nègres » (six fois), « infidèles » (trois fois). Quant à « Mahomet », l'expression « Dieu des infidèles » intervient par quatre fois. Voir José Ángel García Landa, Les Musulmans dans la chanson de geste, Saragosse, Université de Saragosse, 1990, édition électronique, 2004. 
violence meurtrière est une vertu puisqu'ils l'utilisent contre les ennemis de la chrétienté : les Sarrasins.

C'est sur les bases de ce manichéisme que l'on bâtira le monde de l'Orient et de l'Occident. Alain Ruscio dit à propos de La Destruction de Rome que

[1]a sauvagerie des Sarrasins atteint un degré extrême. L'émir fait tuer tous les prisonniers, laïcs et religieux, femmes et jeunes filles. Les Sarrasins se livrent aux pires atrocités, coupant les nez et les lèvres, le poing et l'oreille de leurs victimes innocentes, violant les religieuses [...]. Entrés dans Rome, ils décapitent tous ceux qu'ils rencontrent. Le pape lui-même est décapité dans la basilique de Saint-Pierre ${ }^{3}$.

Cependant, l'Histoire ne cite aucun nom de pape décapité dans la basilique Saint-Pierre ni dans tout autre endroit du monde. Paul Bancourt assure « que le pape est mort de la façon la plus naturelle qui soit ${ }^{4}$ ». L'auteur se demande si tel ou tel acte de barbarie attribué aux Sarrasins n'a pas été, en réalité, commis par des Normands ou des Hongrois.

Mais c'est à partir de ce type de rumeurs engendrées par la peur de connaître et d'approcher l'Autre, de ces rumeurs non démenties voire alimentées ${ }^{5}$ que se construit l'image stéréotypée d'un barbare, d'un ennemi de la religion. La haine succède à la peur de l'Autre et alimentera l'action des croisades.

Ainsi au Moyen Âge, l'habitant de l'Algérie, parce qu'il a participé au rêve d'islamisation du prophète Mohamed, a été pour les Francs lui aussi un Sarrasin. Il était perçu comme noir ce qui, dans la symbolique chrétienne, est synonyme de ténèbres et donc de Satan. Sanguinaire, cruel, violent, laid, fourbe, il était cependant craint et parfois même respecté.

\section{L'Algérien-indigène : un Sarrasin exemplaire}

Telle la reproduction actualisée d'une altérité négative, la haine du Sarrasin, au Moyen Âge, va se déplacer et se fixer sur le Barbaresque. Considéré comme fanatique musulman, ce dernier est qualifié de vicieux (amour immodéré pour les femmes), de hors la loi et de paresseux. Pour les voyageurs de passage, le Sarrasin désigne tout à la fois le Turc, l'Arabe

3. Alain Ruscio, «Des Sarrasins aux Beurs, une vieille méfiance », Le Monde diplomatique [en ligne], février 2004. URL : <http://www.monde-diplomatique.fr/2004/02/RUSCIO/ $10999>$, p. 10.

4. Paul Bancourt, Les Musulmans dans la chanson de geste du Cycle du Roi, Aix-Marseille, Publications de l'université de Provence, 1982, p. 430-515.

5. Voir par exemple la représentation du Sarrasin sur les vitraux des églises. Ce Sarrasin est arabe et/ou musulman.Ses traits sont une caricature de l'image du diable, crasseux, noir et laid. 
et le Maure. Le Turc synonyme de Barbaresque se conduit sur mer comme sur terre comme un despote sanguinaire. Il est craint pour sa cruauté non seulement par les Occidentaux mais aussi par les Arabes et les Maures. Ces derniers, cruels avec les Juifs, sont, eux aussi, des fanatiques musulmans qui, face aux Turcs, deviennent serviles et craintifs.

Ainsi le mot barbare qui aurait dû désigner l'habitant d'un pays, en l'occurrence la Barbarie, prend un sens péjoratif et désigne les Turcs, les Arabes, les Maures et par extension, tout musulman, qui par définition, est « inhumain, d'une grande cruauté ${ }^{6}$ ». Aussi ces témoignages négatifs vontils, par un phénomène de sur-généralisation, alimenter toute une littérature traitant de la course et de l'esclavage, qui donne du «Barbaresque » une image des plus terrifiantes.

\section{Des récits de voyage aux romans coloniaux}

Les récits de voyage sont la première manifestation littéraire en Algérie, comme partout ailleurs dans les colonies. Ils sont le fait de métropolitains venus dans la régence, dans le pays des corsaires, en quête d'informations, d'idées ou par simple curiosité. Ils donnent alors des images pittoresques "plus ou moins idéalisées des colonies ${ }^{7}$ ». Robert Randeau ironise sur cette littérature d'escale aux contours très exotiques :

\footnotetext{
Si le lecteur veut une autre pâture exotique, remplacez le palmier par un manguier, le minaret par un bungalow, le mirage par un récif de corail, la dune par la savane, le djebel par le morne, l'eunuque par le créole, le chibouck par une canne à sucre, la mouquère par une quarteronne; ne changez pas au reste le fond de l'histoire ${ }^{8}$.
}

Le colon opposera « à cette littérature sur l'Algérie faite par des écrivains du dehors ${ }^{9}$ » une littérature faite par des hommes nés dans la colonie : « Voilà ce que l'histoire nous dirait s'il nous venait à l'idée de l'interroger ${ }^{10}$. » $\mathrm{Ce}$

6. Petit Larousse illustré, 1990.

7. Alain Calmés, Le Roman colonial en Algérie, Paris, L'Harmattan, 1984, p. 60.

8. Lucienne Martini et Jean-François Durand, Les Cahiers de la Sielec, $\mathrm{n}^{\circ} 5$ : Romanciers français d'Algérie et société coloniale, 1900-1950. Suivi de Robert Randeau et l'Afrique, Paris, Kailash, 2008, p. 42. Robert Rondeau sera cité à travers ses textes : Les Explorateurs, 1909; L'Aventure sur le Niger, 1913.

9. G. Audissio, L'Algérie littéraire, Paris, Éditions de l'encyclopédie coloniale et maritime, 1948, p. 12.

10. Le croisement de races européennes a donné naissance à une nouvelle race de colons. Louis Bertrand en fait l'apologie dans tous ses romans. Pour lui, seul l'apport de « ces races bordières » redonne de l'énergie aux colons de France « offrant tous les stigmates d'un sang pauvre et comme empoisonné de gaz méphitiques ». De nombreux romans sont écrits à la gloire de ces hommes. Cf. Ferdinand Duchène, Ceux d'Algérie, Paris, éd. des Horizons de France, 1929, p. 8. 
titre de Ferdinand Duchène ${ }^{11}$ annonce tout un programme historique dont les points désignés par le présentatif « voilà », qui est en fait un « jeu de mot» injonctif (vois-là), deviennent les constituants du visible et du lisible.

Les écrits coloniaux justifient alors la mission civilisatrice en montrant les zones colonisées comme dépourvues de toute forme de civilisation. Quelques attributs mélioratifs jadis appliqués à l'Arabe - courageux, noble, cavalier émérite - disparaissent complètement au profit d'autres, diamétralement opposés : fourbe puisqu'il frappe dans le dos, cavalier médiocre puisqu'il monte une " bique ». Certains vocables qu' on retrouve pratiquement dans tous les écrits, et ce depuis les croisades, sont connotés péjorativement comme fanatique, fataliste, sauvage, sanguinaire.

L'image de l'indigène, inférieur à plusieurs niveaux, contraste fortement avec celle de l'Algérien ${ }^{12}$, personnage civilisé, supérieur et mieux placé. Dans ces romans instruits par les politiques, les auteurs comme Duchène dans Mouna, cachir et couscous ou dans Ceux d'Algérie, types et coutume établissent une typologie réduite à trois groupes sociaux : les Algériens, les Juifs et les Arabes. D'autres comme Louis Lecoq dans Pascualette L'Algérien ${ }^{13}$ raconte le parcours héroïque d'un colon, représentant symbolique de l'Algérien, en butte aux forces de la nature et aux vices de l'Arabe. En effet, Pascualette l'Algérien est un personnage typique du roman colonial, un enfant de la troisième génération. Il est d'origine espagnole et a épousé une Française de souche. Les « jeunes arbres » issus de ce mariage sont « de braves petits, qui de leur double ascendance avaient hérité les traits délicats d'une race fine, et des yeux chauds d'Andalousie ${ }^{14} \gg$.

Dans ce récit, l'auteur s'attribue le rôle d'historien : il divulgue un savoir, il donne à voir un représentant d'un groupe social qui a amené la vie à cette terre. Il le résume et l'éclaire tout à la fois. Cependant, le portrait qu'il en fait est à la mesure d'un héros mythique : cet homme famélique se nourrissant à peine, travaillant souvent « vingt heures par jour pendant trois mois ${ }^{15}$ » avec pour but de gagner sa vie dignement. Il se reconnaît aussi par sa croyance religieuse : il est chrétien. La religion chrétienne est un trait d'union entre les membres de ce groupe social. Elle se donne à voir à travers des pratiques religieuses et des superstitions. La plus spectaculaire, importée d'Espagne, est la célébration des fêtes de Pâques. On lui associe « la pâtisserie symbolique chrétienne ${ }^{16} »$ : « la mouna ». Elle concrétise un rite qui «s'impose à la multitude méditerranéenne de l'Algérie avec l'autorité

11. Ferdinand Duchène, Mouna, cachir et couscous, Paris, Albin Michel, p. 245.

12. Le terme d'Algérien désigne celui qui est né de parents européens ou d'origine européenne.

13. Louis Lecoq, Pascualette l'Algérien, Paris, Albin Michel, 1934.

14. Ibid., p. 20.

15. Ibid., p. 46.

16. Ferdinand Duchène, Mouna, cachir et couscous, op. cit., p. 23. 
d'une loi promulguée ${ }^{17}$. » Pendant cette période, tous les Algériens s'unissent pour communier en préparant et en mangeant de la mouna. À cette fête sont conviés quelques Arabes, " une élite intellectuelle musulmane ${ }^{18}$ », et les Juifs qui se sont naturalisés. Pourtant ni le Juif ni l'Arabe ne sont algériens. Mohamed ${ }^{19}$ ne peut être français. Même si Mohamed est ingénieur, avocat, écrivain, un membre de l'élite intellectuelle, l'administration elle-même le qualifiera d' « indigène musulman ». L'Arabe, le musulman ou Mohamed peut être tour à tour, « Mohand » quand il s'agit d'un berbère arabisé, un « mahom $^{20} »$ (diminutif de Mahométan), un « burnous », « un bicot», un « $\operatorname{bic}^{21} »$ (diminutif de bicot).

Cet indigène musulman est brutal, violent et sanguinaire : « Son péché mignon était de couper la tête aux chrétiens ${ }^{22}$. » Il « scie la gorge ${ }^{23}$ » de ses ennemis, « laisse derrière lui des enfants coupés en morceaux ${ }^{24}$ » et maltraite ce qui lui sert de monture. Individualiste et sauvage, il n'a ni le sens de la famille ni celui de la patrie. Il n'existe entre lui et ses congénères « nulle cohé$\operatorname{sion}^{25}$ ». Il faut pour les grouper " prêcher la guerre sainte » : "Abdelkader ne put devenir un grand général que parce qu'il était marabout ${ }^{26}$. 》

Cette catégorisation (bons vs mauvais, indigène $v s$ Algérien, race supérieure $v s$ race inférieur, etc.) se poursuit dans les journaux illustrés tels que Le Petit Parisien ou Le Petit Journal. Les publications à caractère scientifique, les discours politiques, les zoos humains ${ }^{27}$, mais aussi et surtout l'école

17. Ibid., p. 24.

18. Ibid., p. 137.

19. Mohamed, prénom qui sera employé pour désigner - avec une intention dépréciative - tout Arabe.

20. Louis Lecoq, Pascualette l'Algérien, op. cit., p. 46.

21. Ibid., p. 118.

22. Ferdinand Duchêne, Mouna, cachir et couscous, op.cit., p. 46.

23. Ferdinand Duchêne, Ceux d'Algérie, op. cit., p. 48.

24. Ibid., p. 25.

25. Ferdinand Duchêne, Mouna, cachir et couscous, op. cit., p. 130.

26. Ibid.

27. Les zoos humains sont des expositions ethnologiques où des individus exotiques mêlés à des bêtes sauvages étaient montrés en spectacle derrière des grilles ou des enclos à un public avide de distractions. Ces représentations ont été au départ données à des fins purement commerciales. En effet, en 1877, pour redresser sa situation financière désastreuse le directeur du Jardin d'acclimatation de Paris organise « deux spectacles ethnologiques ». Le public de Paris accourt pour découvrir ce que la grande presse a qualifié «d'animaux exotiques accompagnés par des individus non moins singuliers ». Ces représentations se sont multipliées à travers la France sur les places publiques dans les marchés hebdomadaires, dans les foires. Si au début elles ont été données uniquement à des fins commerciales, par la suite elles ont été adaptées à des fins purement politiques. Ce ne sont donc pas les romans coloniaux publiés à quelques exemplaires (entre vingt-sept et trente exemplaires par roman) qui ont permis au Métropolitain d'aller à la rencontre de l'Autre mais bien les zoos humains. Pascal Blanchard, «Entre apothéose et oubli », Le Monde diplomatique, " Polémique sur l'histoire coloniale », juillet-août 2001, p. 41. 
française et coloniale, ont permis la fixation de cette image stéréotypée de l'Autre dans l'imaginaire français.

Si dans les écrits de Duchène, les indigènes sont passifs et vils, ils sont dans les écrits de Louis Bertrand, négligés : de brèves apparitions les présentent sous des traits simplifiés, qui occultent leur identité culturelle. L'écrivain fait le portrait du colonisateur et ne soupçonne pas que le colonisé puisse avoir un destin ${ }^{28}$.

\section{La littérature des indigènes musulmans}

Une première littérature tente, pour exister, d'intégrer et d'assimiler le discours colonial. C'est l'exemple de romans tels que El Euldj captif des barbaresques ${ }^{29}$ et Myriam dans les palmes ${ }^{30}$. Le premier roman reprend les stéréotypes de l'imagerie coloniale en mettant en exergue l'action civilisatrice du colon. Quant au second il raconte l'histoire d'un peuple ignare que la France est venue libérer. Ce discours élogieux, que l'on retrouve dans tous les romans indigènes de l'époque, a, selon Mohamed Ould Cheikh, une fonction bien précise : « essaye[r] tout simplement de faire plaisir ${ }^{31}$. »

Cette littérature d'allégeance reprendra presque la totalité des attributs utilisés dans le discours colonial pour dire le Barbaresque : despote, sanguinaire, violent, etc. Aussi l'éloge vibrant de la France, la critique du « Même » prise en charge par un indigène, produit de l'école coloniale, l'utilisation de tous les modèles stéréotypés utilisés pour donner à voir Mohamed, fonctionnent-ils à une première lecture, comme un démenti aux discours des détracteurs de la France : « qu'il s'agisse de la vision de son espace, de son mode de consommation, de sa culture musulmane le narrateur semble produire un discours de rejet de Soi et d'assimilation à l'Autre ${ }^{32}$. »

Adopter ces modèles stéréotypés de l'Autre, donne l'impression au romancier de réaliser un espace d'entente, la représentation collective figée participe de la doxa ambiante : la grandeur de la France. En la partageant avec lui, il se positionne : il réclame un statut autre que celui de « Mohamed», et fait le vœu d'intégrer la classe des dominants.

Cette stratégie argumentative qui consiste à aller dans le sens de son interlocuteur est adoptée dans Myriam dans les palmes, dès l'avant-propos; elle

28. Alain Cames, «L'idéologie du roman colonial d'expression française en Algérie avant 1914 », vol. I, thèse de doctorat d'État es Lettres, sous la direction du professeur Moïse Le Yaouanc et du professeur Bernard Hue, Université Rennes 2, 1982. En ligne sue le site $<$ www.limag.org $>$.

29. Chukri Khodja, El Euldj captif des barbaresques, Alger, ANEP, 2005.

30. Mohammed Ould Chéikh, Myriam dans les palmes, Oran, Plaza Oran, 1936.

31. Dans l'avant propos de son roman, Ould Cheikh déclare : « J'essaye tout simplement de faire plaisir aux pionniers du rapprochement franco-musulman. En leur dédiant ce modeste travail. »

32. Djaghloul, préface de El Eudj captif des barbaresques, Alger, ANEP, 2005, p. 18. 
procède d'une attitude sociale conditionnée par la situation socio-historique de l'indigène. Ce dernier doit reconnaître et partager les valeurs de l'Autre, ce qui lui permet de transformer les rapports existants entre lui et ses interlocuteurs. Cette transformation procède d'un faire-voir qui consiste à reprendre par écrit certains stéréotypes véhiculés dans les discours coloniaux. Par cet acte, il assimile l'idéologie de la colonisation.

\section{Détruire les stéréotypes comme moyen d'exister}

Dans les années 1950, les œuvres de Dib deviennent autant d'armes de combat qui ont pour fonction première de familiariser le lecteur avec le monde de l'indigène que l'idéologie coloniale avait donné à voir comme étant un monde de barbares qu'il fallait amener à la civilisation.

La trilogie Algérie de Mohamed Dib ${ }^{33}$ tente de déconstruire les stéréotypes ${ }^{34}$ liés à l'Arabe. Les plus anciens et les plus durables sont communs « au contexte de l'énonciation et de la réception ${ }^{35}$ » et ce sont ceux, rappelons-les, du barbare sanguinaire et du Sarrasin cruel.

Dib décrit des hommes et des femmes qui errent dans la rue, donnant à voir la situation sociale à laquelle mène la paupérisation sans cesse grandissante des classes pauvres. La rue (lieu de vie des SDF devenant sous les stigmates de la faim et de l'errance des sans-papiers voire des meurt-de-faim) devient un jour leur lieu de mort. On reconnaît là un topos majeur non seulement du naturalisme, mais aussi du discours édifiant en général ${ }^{36}$. Sur ce lieu commun se greffe tout un jeu d'oppositions stéréotypées : femmes vs hommes, jeunesse $v s$ vieillesse, santé $v s$ maladie, famine $v s$ nourriture, etc.

Les occurrences de cette dernière dichotomie, plus spécifiquement celles du verbe manger, parcourent les romans comme un thème obsessionnel. Aussi, la répétition du vocable pain à travers la trilogie, apparaît comme la topique structurant l'œuvre. Elle forme, par exemple dans le premier roman, sous des dénominations synecdochiques ascendantes, un fil d'Ariane tissant

33. Cette trilogie se compose des romans suivants : La Grande Maison, Paris, Seuil, coll. « Méditérannées », 1952; L’Incendie, Paris, Seuil, coll. « Méditérannées », 1954 et Le Métier à tisser, Paris, Seuil, coll. « Méditérannées », 1957.

34. Jean Louis Dufays, Stéréotype et Lecture, Liège, Mardaga, 1994, p. 81 : « Les stéréotypes dont il est question ici sont évidemment en premier lieu les associations figées du lexique qui font que tel signifiant suggère automatiquement tel(s) signifié(s) : chaque expression, chaque mot véhicule à lui seul un stéréotype. Mais la stéréotypie de la langue ne se limite pas aux unités lexicales. Toutes les associations de mots, toutes les phrases doivent, pour être intelligibles, se conformer à des schèmes sémantiques préexistants, des combinaisons déjà maintes fois réitérées : on ne peut produire et comprendre un discours qu'à la lumière des stéréotypes de la langue. »

35. Ibid., p. 31.

36. La crise mondiale de 1929 a jeté sur les routes des milliers d'hommes, de femmes et d'enfants dont la paupérisation sans cesse grandissante a fait des milliers de morts. 
l'écriture : « un petit bout de pain ${ }^{37}$ », « son croûton ${ }^{38} »$, « une tranche de pain ${ }^{39} »$. Cette parcellisation est évolutive. Elle progresse au rythme du roman en le marquant dès la première phrase du prologue par l'expression « un peu » dans « un peu de ce que tu manges » et en le clôturant, dans la dernière phrase par l'expression miche dans « il tendit la miche à sa mère ${ }^{40} »$. Entre ces deux vocables, les termes et les expressions se multiplient dans une progression quantifiante : " un croûton », un « quignon », pour devenir un « morceau de pain » voire une « tranche de pain ».

Toutes les figures stéréotypées contenant le lexème de la langue française pain sont développées voire données à comprendre à travers de petits drames vécus au quotidien par les personnages. "Avoir peur de manquer de pain ${ }^{41}$ » était leur seule préoccupation. Quand il arrivait qu'il «n'y ait ni pain ni pâtes au logis ${ }^{42} \gg$ les enfants, par la force de l'habitude, parce qu'ils « savaient qu'il n'y avait rien à manger, sans demander d'explication, s'allongeaient sur une couverture, une peau de mouton, par terre, ou à même le dallage, et observaient un silence obstiné ${ }^{43} 》$. En thématisant l'instinct de survie, l'auteur plonge l'Autre dans un passé proche, celui des deux guerres mondiales qui lui ont appris à connaître la faim et la peur, à craindre la mort, et à espérer la vie. Cette stratégie discursive qui consiste dans une relation circulatoire entre un « je/nous » qui « souffre/souffrons » et un « vous » qui « avez souffert», se double d'une fonction émotionnelle car elle mobilise et l'intelligence et la mémoire sensorielle du lecteur. Ce n'est donc pas seulement à sa raison que l'auteur s'adresse mais aussi à son affect. Il veut le toucher et donc le ramener à une époque où cette faim l'avait conduit à la révolte.

La lisibilité du texte tient aussi à la stabilité diachronique de quelques macrostructures sémantiques bien connues dont la principale est l'opposition entre le pauvre et le riche, voire entre le colon et le colonisé.

Le très pauvre, Veste-de-Kaki, « un mioche de rien du tout ", « emmailloté dans une veste de coutil d'été », au " visage pâle et inquiet », au « torse exigu », aux « jambes frêles ${ }^{44}$ », et aux « yeux immenses exprim[ant] une interrogation muette de bête apeurée », est toujours seul à l'écart; il n'existe pas, il n'a pas d'identité aux yeux du nanti Berri qui «bâfrait ${ }^{45}$ ", s' « empiffr(ait) » et dont le signe extérieur, ostentatoire de richesse, est le « sac de cuir, à broderie d'argent et or ». Cet enfant de riche jouissait d'un

37. Mohamed Dib, La Grande Maison, op. cit., p. 7.

38. Ibid.

39. Ibid.

40. Ibid., p. 190. C'est moi qui souligne.

41. "Avoir peur de manquer de pain » signifie être inquiet de l'avenir.

42. " Il n'y a ni pain ni pâte au logis » signifie il n'y a plus rien à manger.

43. Mohamed Dib, La Grande Maison, op. cit., p. 57-58.

44. Ibid., p. 12.

45. Ibid., p. 12-14. 
grand respect. On le protégeait ${ }^{46}$, on l'entourait, on le servait et on se contentait de ramasser les quelques miettes qu'il laissait tomber.

Driss s'adossait à un mur [...], et bâfrait posément. De temps en temps, quelqu'un se baissait pour ramasser des miettes. Il avait un camarade qui se chargeait de son sac de cuir, à broderie d'argent et d'or, à la sortie de l'école. D'autres, quand approchait l'heure d'entrer en classe, allaient le chercher et lui tenaient compagnie en chemin. Ils ne se séparaient de lui que lorsque la cloche sonnait. C'était à qui se mettrait à ses côtés, et qui poserait une main sur son épaule ${ }^{47}$.

Le texte gagne en lisibilité grâce à l'utilisation d'une partie du code stylistique de son lecteur. La permanence d'une partie de ce code permet aux lecteurs d'identifier un certain nombre d'expressions figées ou semi-figées comme « mains tendues », « tromper notre faim », « joie angélique », « craintes sans bornes », « bête apeurée », « propres à rien », ou encore « incurable comme une maladie des tropiques ». Certaines expressions charrient la référence coloniale : « j'ai travaillé comme un nègre ${ }^{48} »$; d'autres s'affichent comme idéologiques : « Les hommes ne sont pas faits pour la maison. » Ces images, réitérées par leur caractère conventionnel et familier, ont pour mission de représenter la base de la vraisemblance par excellence. Elles assurent le lien entre la réalité de l'indigène et le savoir du lecteur potentiel, en l'occurrence 1'Autre. Mais certaines d'entre elles subissent des transformations par un travail de synonymie. Ainsi, dans L'Incendie, « la route serpente » devient « la route ondulait » ou encore « la route ondulait, déroulait ses sinuosités puis disparaissait dans le lointain ${ }^{49} \gg$. La notion de mouvement, de progression reptilienne que suggère l'utilisation du lexème serpent devient par le jeu de la synonymie (ondulait, déroulait, disparaissait) une avancée qui se pourrait être la voie prometteuse que tout être menacé se doit d'emprunter. Le serpent tel le scorpion peut mordre et tuer de son venin, de sa colère, tout individu qui se trouve sur son chemin. D'autres lexèmes expriment le drame du colonisé diminué par la misère et la maladie. Spolié de ses terres et de ses biens, il n'aura d'autres choix que la mendicité ou la servitude. Citons à titre d'exemple les habitants de Dar-Sbitar que la misère, le désespoir et l'humidité rongent : « la cave bourdonnait d'une excitation furtive $[\ldots]$ désespérée ${ }^{50}$. »

Parallèlement à cette appropriation du code stylistique de « l'Autre », s'opère une intrusion d'images traduites littéralement de l'arabe : « Vous

46. Ibid., p. 12-13 : « Omar ni personne n'osait toucher, sans encourir de grands châtiments de la main des maîtres, les quelques fils de négociants, de propriétaire, de fonctionnaires qui fréquentaient l'école. »

47. Ibid., p. 12-14.

48. Mohamed Dib, L'Incendie, op. cit;, 1954, p. 144.

49. Ibid.

50. Mohamed Dib, Le Métier à tisser, op. cit, 1957, p. 77. 
vous êtes fixés sur moi comme des sangsues »; «Quand tes pieds ne t'ont plus porté il t'a jeté comme une ordure »; " Maudits soient les pères et mères de ces cardons ${ }^{51} » ;$ « Mon destin de malheur ${ }^{52} »$.

En parasitant le code stylistique, en faisant cohabiter deux univers de références culturelles différents, l'auteur instaure un bizutage du code linguistique de « l'Autre ». Il en parodie les topos et crée, par voie de conséquence, un pacte de lecture à travers des manœuvres poétiques de déconstruction de la topique usuelle du français. Mais, la langue, ainsi travaillée, devient polyphonique et le lecteur français se trouve pris entre familiarité et étrangeté : ce sont déjà les prémisses d'une écriture de l'entre-deux. Certaines figures traduites de l'arabe ont une résonance étrange, même pour un lecteur indigène. Ainsi, " La mort pour nous est une couverture d'or ${ }^{53}$ » est une expression figée qui n'a de sens que pour un lecteur autochtone.

Dans La Grande Maison, la communauté indigène vit en marge des dominants. Ces derniers ne sont jamais caractérisés d'une manière différentielle. Pour les nommer, le dominant utilisera le pronom personnel « ils ». Ainsi, par exemple, quand le personnage nommé Commandar constate : « ils tuent, ils chassent les nôtres de la lumière ${ }^{54} »$, son interlocuteur sait qu'il s'agit des colons. L'intrusion de ces derniers dans l'univers des dominés est sporadique mais suffisante pour dire le dictat qu'ils font peser sur eux : Gonzalés est un des patrons qui exploitent la force de travail des femmes telle Aïni en échange de quelques sous. L'intrusion de la police dans Dar Sbitar en est un autre exemple; elle est une force répressive au service du colonat. Cependant, la mémoire convoquée par le personnage Hamid Serradj est un contre-discours qui permet aux hommes de prendre conscience de leur existence en leur rappelant qu'ils ont un passé et une histoire :

Oum El Kheir te dira que son père était un grand guerrier, un grand cavalier, un sage plus sage que tous les autres dont la justice et la bonté, mais surtout la bravoure étaient plus grandes que chez les autres hommes de la tribu et tout cela n'était rien encore; parce que son grand père était plus que cela c'était un homme-roi ${ }^{55}$.

Par le récit de ces petits drames qui font le quotidien de l'indigène, l'auteur donne à voir autrement l'indigène mais aussi le colon. Luttant pour survivre l'indigène se montre patient mais sa souffrance le pousse à la révolte. En convoquant son passé il a compris qu'il n'était pas tel que les colons le décrivaient et il s'est uni au "Même ». Cette force nouvelle va, pour se défendre, utiliser les mêmes armes que l'occupant.

51. Mohamed Dib, La Grande Maison, op. cit., p. 11.

52. Ibid., p. 36.

53. Ibid., p. 84.

54. Mohamed Dib, L'Incendie, op. cit., p. 141.

55. Ibid. 


\section{Au lendemain de l'indépendance}

Avec l'indépendance de l'Algérie, l'indigène change de statut. Il devient un Algérien libre qui va avoir Une paix à vivre ${ }^{56}$. Cependant, très rapidement «le fleuve» va être «détourné ${ }^{57}$ » puisque, comme le dira Ferhat Abbes, leur indépendance va leur être confisquée. Trente ans après son indépendance, l'Algérie bascule dans le terrorisme et l'écriture se réalise dans l'urgence, réactivant certains stéréotypes de jadis comme explication de l'actualité des années 1990.

Déjà dans Une paix à vivre de Rachid Mimouni, de jeunes normaliens protestent contre le coup d'État de Boumediene. Emprisonnés, on les assignera au silence ou à la torture. Muselés, ils vont se disperser. Cette œuvre, tout comme l'Algérie, est exemplaire de ce moment à deux temps qui a caractérisé l'évolution de nombreux pays du tiers-monde après la décolonisation :

- un temps où, portés par l'euphorie due à la guerre de libération et aux discours officiels, les Algériens, et par ricochet les personnages du roman de Rachid Mimouni, vont tenter de vivre la paix;

- un second temps dysphorique, marqué par des difficultés qui vont aller crescendo et qui vont amener peu à peu les Algériens à prendre conscience, non seulement que leur indépendance leur a été confisquée, mais qu'ils ont de la peine à vivre ${ }^{58}$, une peine à respirer qui va les mener peu à peu vers une forme de révolte qui va les diviser en deux mondes cohabitant dans un même espace.

Marie Virolle écrira dans la postface du roman Peurs et Mensonges d'Aissa Khelladi publié dans la revue Algérie Littérature/Action : « C'est un constat d'histoire. L'Algérie est un gouffre bouillonnant de contradictions politiques, économiques et sociales ${ }^{59}$.

Ce roman avertit le lecteur, dès le paratexte, « qu'il a affaire à un discours sans autre règle que celle de transcrire scrupuleusement le réel ${ }^{60} »$. L'établissement dès le départ de ce protocole de lecture, ou plus exactement son insistance à ne pas libérer le texte fictif, représente pour lui un moyen privilégié d'écrire la vrai-semblance.

Dans la revue Algérie Littérature/Action, l'objet de ce discours est résumé dans le titre inaugural, Peurs et Mensonges et sous-tend toute la production. Il peut s'énoncer comme suit : la violence en Algérie ou la tragédie algérienne.

56. Rachid Mimouni, Une paix à vivre, Paris, Stock, 1983.

57. Cf. Rachid Mimouni, Le Fleuve détourné, Paris, Stock, 2000.

58. Rachid Mimouni, Une peine à vivre, Paris, Stock, 1994.

59. Marie Virolle, Algérie Littérature/Action (Al/Lit/Act), $\mathrm{n}^{\circ} 1$, Peurs et Mensonges, Paris, Marsa, 1996, p. 173.

60. Présentation dans Tzvetan Todorov (éd.), Littérature et réalité, Paris, Seuil, 1982, p. 7. 
Cette violence se situe dans un champ idéologique qui la surdétermine, et cela quelles que soient les intentions exactes des écrivants de la revue; elle a fait et fait encore l'objet de nombreux reportages, de nombreuses émissions et de nombreuses pages à la une des presses algérienne et française. Cependant, la revue en propose un traitement particulier. Elle se propose de donner à voir et à comprendre l'Algérie et l'Algérien par ses attributs, ceux du Sarrasin qu'il fut : violent et sanguinaire, adepte d'une religion obscure et terroriste.

Dans l'ensemble des romans publiés dans la revue, les personnages ont une vision manichéenne de l'univers romanesque. Les mauvais seraient les « frères musulmans ${ }^{61}$ » symbolisant le destin et les bons, toutes les victimes de ces derniers. Ainsi, dans L'Étoile d'Alger:

Jeté de l'école très tôt, Baiza a viré mauvais. D'abord l'alcool solide, ensuite tout : kif, amphétamines, chaos technique. Trois fois repris de justice pour agressions à l'arme blanche, deux fois six mois de taule. Véritable vipère, déguisé depuis peu sous les traits de l'islam ${ }^{62}$.

\section{Ou dans Meriem ou la Déchirure}

— Tu te souviens de « Nouh ezzouffri »...,

— Ce n'est pas celui qui est sorti de prison cet été...? Lance Petit Moh incertain. - Tout a fait! Eh bien, il a été pris en main par les gars de la mosquée « Es Sunna ».

- Et alors, précise le bavard, lui qui était un pilier de bar, le voilà qui ne quitte plus la mosquée. Il s'est laissé pousser la barbe et ne s'habille plus qu'en Kamis ${ }^{63}$.

Les Frères musulmans sont omniprésents dans l'ensemble des romans. Ils sont perçus comme violents. Ils sont ceux par qui la mort arrive avec son cortège de souffrances et de larmes. On en a peur car ils représentent une menace pour l'ordre établi. Ainsi rien n'a changé. L'Arabe de Duchène ou celui de Camus deviennent le « frère musulman » ou encore le « barbu ». Car n'oublions pas que Meursault, l'étranger de Camus, a tué parce qu'il avait peur de «l'Arabe ». Sa peur, une peur ancestrale, l'a mené malgré lui au crime.

On assiste, dans cette littérature des années 1990, à une actualisation du vocable Sarrasin : il désigne désormais les Frères musulmans puis le musulman tout court.

61. Les Frères musulmans appartiennent à une confrérie religieuse héritée de l'Égypte de Djamal Abdelnacer. Adeptes d'une vision très extrémiste de la religion musulmane, ils seront perçus comme fanatiques et à l'origine des mouvements terroristes des années 1990.

62. Azziz Chaouki, L'Étoile d'Alger, Algérie Littérature/Action, n 14, Paris, Marsa, 1999, p. 56.

63. Abdenour Nouiri, Meriem ou la Déchirure, Algérie Littérature/Action, n $31-32$, Paris, Marsa, mai-juin,1999, p. 26. 
Cette vision stéréotypée du Frère musulman ou du «barbu » dans la revue coexiste avec une autre, celle des victimes que sont les intellectuels de culture française. Ce sont :

- les journalistes (Samir Kalder dans Peurs et Mensonges ${ }^{64}$ et Meriem dans Le Premier Jour d'éternité $\left.{ }^{65}\right)$;

- les lycéennes ou étudiantes (Nadia dans Au commencent était la mer ${ }^{66}$, Warda dans Sang et Jasmin ${ }^{67}$ );

- ou les enseignantes (Malika Ryane dans Chroniques de l'impure) pour ne citer que ceux-là.

Chaque roman permet à un personnage d'exprimer ses sentiments, ses convictions et ses problèmes. Sa parole est largement encadrée par le récit. L'intrigue est l'histoire d'un personnage coincé par le temps (le temps présent) et par l'espace (l'Algérie). Son histoire rencontre l'histoire de l'Algérie des années 1990. C'est un personnage tragique dont l'histoire ne peut se dissocier de celle de son pays, l'Algérie. Chaque roman abordé est un drame sombre parcouru par une sorte de « thrène ${ }^{68}$ » pour les défunts. « La souffrance, le deuil, les larmes » seraient ce que Jean-Marie Domenach appelé « le matériau ordinaire de la tragédie ${ }^{69} »$.

La description de l'Algérien est proche de celle du Sarrasin d'autrefois. De nouveaux qualifiants viennent se greffer sur les anciens : l'Algérien est toujours sale et vit dans la promiscuité. C'est un fainéant : avant il était accroupi dos au mur, aujourd'hui il est toujours dos au mur mais debout (un hittiste ${ }^{70}$ ). C'est aussi un voleur, un menteur et un sauvage. Avant il portait la gandoura, aujourd'hui il porte le kamis. Il était une menace dans le passé, mais une menace contrôlée. Aujourd'hui c'est un fanatique, un sanguinaire assoiffé de sang, "une bête féroce », mais surtout il peut, tel un monstre qui hante la littérature, prendre le visage de l'innocence.

Exploitant une émotion aussi primaire que la peur de l'étranger, les fondateurs de la revue Algérie Littérature/Action, ont choisi de donner une image paroxysmique de l'Algérien. Les romanciers choisis ont puisé dans un stock préexistant de représentations collectives que nous retrouvons dans les

64. Amine Touati, Peurs et Mensonges, Algérie Littérature/Action, n ${ }^{\circ}$, Paris, Marsa, 1996. 65. Ghania Hammadou, Le Premier Jour d'éternité, Algérie Littérature/Action, Algérie Littérature/Action, $\mathrm{n}^{\circ}$ 12-13, Paris, Marsa, 1997.

66. Maïssa Bey, Au commencement était la mer, Algérie Littérature/Action, ${ }^{\circ} 5$, Paris, Marsa, 1996.

67. Leila Hamouténe, Sang et Jasmin, Algérie Littérature/Action, n43-44, Paris, Marsa, 1996.

68. Terme utilisé par Marie Virolle dans la postface du roman Le Premier Jour d'éternité.

69. Jean-Marie Domenach, "Résurrection de la tragédie », Esprit, numéro spécial, mai 1965, p. 98.

70. Abdenour Nouiri, Meriem ou la déchirure, Paris, Marsa, 1997. Revues n 31-32. Les hittistes, néologisme dérivé de l'arabe « hit », eux qui, à longueur de journées, s'appuyaient contre les murs. 
textes de la période dite « algérianiste », dans ceux de « l'école d'Alger » et dans tous les journaux de l'époque. Ce choix obéit à une politique éditoriale qui vend une littérature algérienne d'expression française à un public français curieux de savoir « qui tue qui » dans cette Algérie des années 1990. Aussi vont-ils, en se basant sur un " déjà vu », sur un « déjà entendu », réexploiter le stéréotype de l'Arabe.

Cette reconstruction du monde dans les romans publiés dans la revue est une argumentation par l'exemple, fondée sur le passage d'un cas particulier (l'histoire d'un personnage) à une règle générale, que la revue aide le lecteur à réaliser. D'une part, parce qu'elle vient conforter une idée préconçue que se fait déjà le lecteur de l'Algérie et de l'Algérien, d'autre part parce qu'elle est présentée dans le paratexte (postface, préface et entretien), non comme vraisemblable, mais comme vraie. Aussi le lecteur ne sera difficile ni à persuader ni à convaincre.

\section{Conclusion}

Civiliser l'Autre en luttant contre sa nature animale a été présenté par la France, à travers ses discours politiques et ses médias, comme une mission d'humanisation, une lutte noble pour éradiquer le mal incarné par l'Arabe, élément dégénératif de la société coloniale. En le définissant comme tel, elle exclut la majorité d'entre eux et condamne quelques-uns à poursuivre un rêve, le rêve de se faire entendre pour accéder un jour au monde de l'Autre. Ainsi, la stéréotypie n'a été pendant la colonisation qu'un instrument qui a servi à diviser l'Algérie en deux clans : le clan des civilisés et le clan d'une sous-humanité.

La littérature de Dib et de toute la génération de l'indépendance tentera d'établir le contact avec l'Autre, curieux de lire comment la vérité se dédouble et devient différente selon que l'on soit dominé ou dominant. Ayant eu à subir, en tant que colonisé, une image déformée de lui-même et des siens, il va pour la rejeter, donner à voir la misère grandissante et ce qui les pousse à la révolte nourrie du désir d'arracher leur liberté.

La production de l'après-indépendance se retrouve vite dans les années 1980 et 1990 commandée par l'actualité, celle de la violence en Algérie.

Le discours manichéen du colon est repris par certains auteurs, ceux précisément que publie la revue Algérie Littérature/Action, faisant de l'islam une terreur et de l'arabe un genre à part. Cette stigmatisation est dangereuse puisqu'elle perturbe les systèmes de représentation de soi et de l'Autre.

Fatima Zohra Lalaoui-Chiali Université d'Oran (Algérie), chercheuse au LASELDI (Besançon) 